\title{
Why are women with obesity more likely to develop breast cancer
}

\author{
Sheng-I Chen ${ }^{1}$ \& Chia-Chien Hsieh*,2 \\ ${ }^{1}$ Department of Industrial Engineering \& Management, National Chiao Tung University, Hsinchu, Taiwan \\ ${ }^{2}$ Department of Human Development \& Family Studies, National Taiwan Normal University, Taipei, Taiwan \\ *Author for correspondence: Tel.: +886 27734 1439; Fax: +886 22363 9635; hsieh@ntnu.edu.tw
}

"In obese adipose tissue, multiple immune cells extensively infiltrate adipose tissue and this results in the characteristic continuous chronic inflammation, which is the major factor influencing breast tumor progression."

First draft submitted: 10 February 2018; Accepted for publication: 16 February 2018; Published online: 25 June 2018

Keywords: obesity $\bullet$ tumor microenvironment $\bullet$ women

\section{Epidemiological studies on obesity \& breast cancer}

Obesity is a critical medical condition originating from abnormal or excess adipose tissues, causing physiological and pathological problems. The global prevalence of obesity has increased rapidly in the past decades. Furthermore, it is well known that obesity is associated with other metabolic diseases, such as type II diabetes, cardiovascular diseases and several types of cancer. Epidemiological studies showed a close link between obesity and cancer development [1]. Excess adipose tissue might contribute to the development of malignancy in organs such as the endometrium, breast, esophagus, liver, colon and ovary [2]. Abnormal obesity is a major factor contributing to breast cancer development. Based on this evidence, a scientific review summarized that obesity is considered to increase the risk of poor outcomes of breast cancer regarding recurrence and mortality by $35-40 \%$ [3].

The World Cancer Research Fund (WCRF) International has reported that higher incidence of breast cancer in postmenopausal patients is attributed to obesity, whereas obesity is observed to reduce the risk of breast cancer in premenopausal women [4]. This conflicting finding is possibly due to different sources of hormonal drivers influencing cellular physiology in the breast tissue [5]. The hormone estrogen is secreted by the ovaries in premenopausal women, while the primary source of estrogen includes adipocytes in postmenopausal women, which was positively correlated with increase in the risk of breast cancer, owing to the effect of aromatase [4,5]. Moreover, certain studies showed that obesity promotes breast cancer progression irrespective of menopause. Recently, a meta-analysis proved that central obesity is associated with modestly increased risks of breast cancer, independent of menopause [6].

\section{Obesity-related breast tumor microenvironment}

Obesity-associated low-grade inflammation is widely accepted as an important process in cancer pathogenesis [7]. Excessive fat accumulation in obesity causes the development of a chronic low-grade inflammatory environment as well as the secretion of numerous adipokines and derived mediators, including proinflammatory cytokines, insulin, IGF-1 and IGF-1 receptor, and increase in estrogen synthesis by adipocytes contributes to the promotion of tumor migration and invasion [2,8]. This inflammation is proposed as an important mechanism connecting obesity with its metabolic complications, such as type II diabetes mellitus (DM), insulin resistance, atherosclerosis and several cancers [9]. More specifically, M1 macrophages infiltrating the adipose tissue increase the production of proinflammatory cytokines, such as MCP-1, IL-6 and so on $[9,10]$. Moreover, adipokines are closely linked to obesity and they play roles in carcinogenesis. The predominant adipokine, leptin is strongly related to obesity; cross talks between inflammatory cytokines and growth factors in breast cancer have been proposed to be essential for tumor development and angiogenesis [11]. The other possible mechanism for obesity-related breast cancer is adipose-tissue hypoxia mediated by HIF- $1 \alpha$, whose activation influences immune cell infiltration, generating the

Future Medicine 
low-grade inflammatory state [12]. Additionally, HIF activation affects the growth and progression of breast cancer by downregulated the estrogen receptor (ER) $\alpha$ and inducing signaling transduction causing tumor angiogenesis [12].

Tumorigenesis involves the regular communication between tumors cells and the neighboring stromal cells including adipocytes, fibroblasts and immune cells in this microenvironment. In obese adipose tissue, multiple immune cells extensively infiltrate adipose tissue and this results in the characteristic continuous chronic inflammation, which is the major factor influencing breast tumor progression $[9,10,13]$. Interestingly, tumor-associated macrophage resembles M2 macrophage triggered immune suppression [10,13]. Additionally, the tumor microenvironment comprises the extracellular matrix, soluble factors and signaling molecules promoting carcinogenesis and evading the immune response. For instance, obesity generates the hypoxic microenvironment, causing the expression modification of genes related to angiogenesis, cell proliferation and apoptosis and ultimately promotes cancer development [12]. Moreover, obesity is frequently accompanied by abnormally high plasma cholesterol levels, which additionally accelerate and exacerbate tumor progression and aggressiveness [14].

\section{Relations between obesity \& estrogen in breast cancer}

Breast cancer is classified into two subtypes: ER-positive (+) type and ER-negative (-) type depending on ER abundance within cells [5]. Approximately $70-80 \%$ of breast cancers are estrogen-sensitive and clinical evidence shows that $\mathrm{ER}(+)$ cancer responds to antiestrogen therapy and has better prognosis, whereas $\mathrm{ER}(-)$ cancer is more aggressive and resistant to treatment [15]. Epidemiological studies prove that obese women have significantly higher bioavailable levels of estrogen and testosterone than women of normal weight [16]. Obesity has been revealed to alter hormone production pathways and thus, enhances aromatase activity in adipocytes to synthesize more estrogen, subsequently promoting carcinogenesis.

Therefore, high estrogen level due to aromatase hyperactivity in adipose tissue has been proposed to promote breast cancer development [15]. Aromatase plays a key role in estrogen synthesis by hormone transformation, and it is synthesized in various tissues, especially undifferentiated adipocytes and adipose fibroblasts [17]. Breast cancer cells include adipose fibroblasts, immune cells and malignant epithelial cells, stimulating aromatase expression and estrogen synthesis in this microenvironment. Aromatase expression is abnormally high in the obese breast with predisposition to neoplasia and hyperplasia in the breast [17]. Therefore, patients with breast cancer are treated with hormone deprivation therapy, such as with aromatase inhibitors. However, it is less effective in obese women than in women of normal weight and additionally, this effect was shown in an obese nude mouse model [18], suggesting that obesity induces resistance of breast tumors to aromatase inhibitor treatment, which might affect cancer prognosis. In summary, interventions, including life style changes, exercise and dietary habits may alter adipose tissue inflammation and estrogen synthesis with beneficial effects on breast cancer risk [19].

Accumulating evidence has shown that obesity gives rise to the high levels of estrogen, proinflammatory cytokines, adipokines and insulin-related factors, inducing hypercholesterolemia and oxidative stress, resulting in breast cancer progression [15]. However, the exact connection remains unknown; to understand the mechanistic link between obesity and breast cancer is a critical need for clarifying targets and strategies to neutralize these procarcinogenic effects of obesity.

\section{Data analytics for identifying risks of breast cancer}

Experiment-based studies are usually conducted on a microenvironment. These approaches ignore the confounding effect of obesity among other risk factors. At a macro level, researchers use experimental and clinical data to examine multiple demographic characteristics of obesity related to breast cancer. A clinical trial was conducted, showing that postmenopausal women with BMI $>35 \mathrm{~kg} / \mathrm{m}^{2}$ at US clinical centers were more likely to develop invasive breast cancer [20]. Recently, Park et al. investigated the risks of metabolic disorder and obesity phenotypes in breast cancer participants 35-74 years in age [21]. A comprehensive review on application of meta-analysis to BMI and breast cancer was performed [5,6].

In recent years, attention has been focused on understanding the cause of cancers based on genomic characteristics. Michailiduo et al. performed a genome-wide association study combined with odds ratio estimates and standard error to identify susceptibility loci for breast cancer [22]. These recent advancements have enabled the emerging shift to cancer treatment by precisely identifying individual risks and guiding clinical decisions. With such a paradigm, oncologists can use the unique genetic characteristics of each patient and develop the most appropriate preventive strategy or treat diseases at early stages. A general framework of genome-based risk assessment for the diagnosis and 
prognosis of cancer is available in an early report by Ginsburgh et al. [23]. Furthermore, interested readers may refer to the comprehensive review on genetically guided personalized medicine for guidance on clinical decisions [24].

Important questions as to how clinically meaningful data can be transformed into intelligence remain unanswered. Data mining is an important approach to obtain valuable insight from raw data. As a practical consideration in healthcare, these techniques are often used to cluster different types of patients appropriately to collect data on their medical, physical and dietary statuses. The information can assist the development of treatment plans and arrangement of long-term follow-up care. Researchers have applied support vector machines and naive Bayes classifiers based on information on bodyweight and obesity comorbidities to classify individuals into obesity, overweight, normal and underweight categories. Obese patients are further categorized into morbid, severe and moderate obesity groups [25]. Another study used association rules and neural networks to establish the relationship between breast cancer and different risk factors, such as obesity, menstrual cycle, disease history and age [26]. The integration of electronic patient records and data mining has accelerated progress in precision medicine. A recent study has begun to use structured formats of breast imaging examinations and data mining techniques to improve the detection and screening of breast cancer [27]. Others have integrated text mining, data mining and network analysis to identify genetic biomarkers of breast cancer [28]. These different research topics regarding the use of distinct data sets have prompted scholars to investigate breast cancer whereby people are almost likely to develop diseases.

In this article, we review multidisciplinary research to understand the relation between obesity and breast cancer and advancements in data analytics for supporting the use of information in taking health-related decisions as well. Our review reflects the need of both fundamental research and analytical tools for the dissemination of laboratory findings and subsequently, convert information into decisions.

Financial \& competing interests disclosure

The authors acknowledge the funding from Ministry of Science and Technology, Taiwan (MOST 106-2221-E-009-114-MY2 and MOST 106-2311-B-003-005-MY3). The authors have no other relevant affiliations or financial involvement with any organization or entity with a financial interest in or financial conflict with the subject matter or materials discussed in the manuscript apart from those disclosed.

No writing assistance was utilized in the production of this manuscript.

\section{Open access}

This work is licensed under the Attribution-NonCommercial-NoDerivatives 4.0 Unported License. To view a copy of this license, visit http://creativecommons.org/licenses/by-nc-nd/4.0/

\section{References}

1. National Cancer Institute (NCI). NIH, USA (2018). www.cancer.gov/about-cancer/causes-prevention/risk/obesity/obesity-fact-sheet

2. Louie SM, Roberts LS, Nomura DK. Mechanisms linking obesity and cancer. Biochim. Biophys. Acta 1831(10), 1499-1508 (2013).

3. Jiralerspong S, Goodwin PJ. Obesity and breast cancer prognosis: evidence, challenges, and opportunities. J. Clin. Oncol. 34(35), 4203-4216 (2016).

4. World Cancer Research Fund (WCRF)/American Institute for Cancer Research (AICR). Chapter 7: Breast. In: Food, Nutrition, Physical Activity, and the Prevention of Cancer: a Global Perspective. American Institute for Cancer Research, DC,

USA, 289-295 (2007). www.aicr.org/assets/docs/pdf/reports/Second_Expert_Report.pdf

5. Byers T, Sedjo RL. Body fatness as a cause of cancer: epidemiologic clues to biologic mechanisms. Endocr. Relat. Cancer 22(3), R125-R134 (2015).

6. Chen GC, Chen SJ, Zhang R et al. Central obesity and risks of pre- and postmenopausal breast cancer: a dose-response meta-analysis of prospective studies. Obes. Rev. 17(11), 1167-1177 (2016).

7. Crespi E, Bottai G, Santarpia L. Role of inflammation in obesity-related breast cancer. Curr. Opin. Pharmacol. 31, 114-122 (2016).

8. Law JH, Habibi G, Hu K, Masoudi H et al. Phosphorylated insulin-like growth factor-i/insulin receptor is present in all breast cancer subtypes and is related to poor survival. Cancer Res. 68(24), 10238-10246 (2008).

9. Sundaram S, Johnson AR, Makowski L. Obesity, metabolism and the microenvironment: links to cancer. J. Carcinog. 12, 19 (2013).

10. Gordon S, Martinez FO. Alternative activation of macrophages: mechanism and functions. Immunity 32, 593-604 (2010).

11. Newman G, Gonzalez-Perez RR. Leptin-cytokine crosstalk in breast cancer. Mol. Cell Endocrinol. 382(1), 570-582 (2014).

12. Rausch LK, Netzer NC, Hoegel J et al.The linkage between breast cancer, hypoxia, and adipose tissue. Front. Oncol. 7, 211 (2017). 
13. Hsieh CC, Peng SH, Chou MJ. Obesity enhances carcinogen 7,12-Dimethylbenz [a] anthracene-induced tumorigenesisin vitro and in vivo. Food Chem. Toxicol. 110, 156-164 (2017).

14. Engin A. Obesity-associated breast cancer: analysis of risk factors. Adv. Exp. Med. Biol. 960, 571-606 (2017).

15. Cuzick J. Chemoprevention of breast cancer. Breast Cancer 15(1), 10-16 (2008).

16. McTiernan A, Rajan KB, Tworoger SS et al. Adiposity and sex hormones in postmenopausal breast cancer survivors. J. Clin. Oncol. 21(10), 1961-1966 (2003).

17. Bulun SE, Chen D, Moy I et al. Aromatase, breast cancer and obesity: a complex interaction. Trends Endocrinol. Metab. 23(2), 83-89 (2012).

18. Schech A, Yu S, Goloubeva $\mathrm{O}$ et al. A nude mouse model of obesity to study the mechanisms of resistance to aromatase inhibitors. Endocr. Relat. Cancer 22(4), 645-656 (2015).

19. Zahid H, Simpson ER, Brown KA. Inflammation, dysregulated metabolism and aromatase in obesity and breast cancer. Curr. Opin. Pharmacol. 31, 90-96 (2016).

20. Neuhouser ML, Aragaki AK, Prentice RL et al. Overweight, obesity and postmenopausal invasive breast cancer risk. JAMA Oncology 1(5), 611-621 (2015).

21. Park YMM, White AJ, Nichols HB et al. The association between metabolic health, obesity phenotype and the risk of breast cancer. Int. J. Cancer 140(12), 2657-2666 (2017).

22. Michailidou K, Beesley J, Lindstrom S et al. Genome-wide association analysis of more than 120,000 individuals identifies 15 new susceptibility loci for breast cancer. Nat. Genet. 47(4), 373-380 (2015).

23. Ginsburg GS, Willard HF. Genomic and personalized medicine: foundations and applications. Transl. Res. 154(6), 277-287 (2009).

24. Welch BM, Kawamoto K. Clinical decision support for genetically guided personalized medicine: a systematic review. J. Am. Med. Inf. Assoc. 20(2), 388-400 (2012).

25. Figueroa RL, Flores CA. Extracting information from electronic medical records to identify the obesity status of a patient based on comorbidities and bodyweight measures. J. Med. Syst. 40(191), 1-9 (2016).

26. Karabatak M, Ince C. An expert system for detection of breast cancer based on association rules and neural network. Expert Syst. Appl. 36, 3465-3469 (2009).

27. Margolies LR, Pandey G, Horowitz ER et al. Breast imaging in the era of big data: structured reporting and data mining. Am. J. Roentgenol. 206(2), 259-264 (2016).

28. Jurca G, Addam O, Aksac A et al. Integrating text mining, data mining, and network analysis for identifying genetic breast cancer trends. BMC Res. Notes 9, 236 (2016). 